\title{
NATIONAL EXTERNAL QUALITY ASSURANCE SCHEME FOR HIV TESTING USING DRIED BLOOD SPOT: A FEASIBILITY STUDY
}

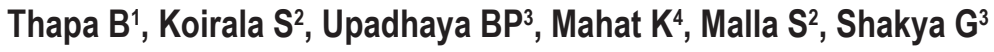 \\ 1 Kathmandu Medical College, Genesis Laboratory and Research, Communicable Disease Poly Clinic and Research \\ Centre, Kathmandu, Nepal, \\ 1,2 UNDP, Kathmandu, Nepal, \\ 3 National Public Health Laboratory, Kathmandu, Nepal, \\ 4 Genesis Laboratory and Research, Kathmandu, Nepal
}

\begin{abstract}
Introduction: Dried Blood Spot (DBS) for National External Quality Assurance Scheme (NEQAS) is becoming popular in resource limited and geographically constrained settings. The feasibility of using DBS for EQAS for HIV testing in Nepal is studied.

Methodology: National Public Health Laboratory (NPHL) and five Voluntary Counseling and Testing Centers (VCT) located at the Mid-Western and Central region of Nepal were chosen as the organizing and participating laboratories, respectively. HIV tests were performed on 35 samples using rapid test kits following the National algorithm. Two sets of samples were blotted on a DBS card, dried, packed, stored and posted to NPHL using normal postal delivery. The eluted DBS were tested by rapid test and ELISA and results were compared.
\end{abstract}

Results: All DBS cards were received in a good condition. The rapid test result obtained at VCT and NPHL was $100 \%$ concordant. By ELISA, $32(91.4 \%)$ and $3(8.6 \%)$ samples were concordant and discordant, respectively. The sensitivity and specificity of the rapid test were $88.9 \%$ and $94.0 \%$, respectively. The DBS was stable with mean transportation time of 2.25 days and storage time of one month.

Conclusion: DBS is an easy, simple, cost effective and a dynamic platform for blood collection in a country like Nepal and can be considered for implementation for NEQAS for HIV testing.

Key words: Dried Blood Spot, External Quality Assurance Scheme, HIV Testing, Nepal

\section{INTRODUCTION}

The diagnosis of HIV relies on accuracy of HIV testing laboratory services in the country and accuracy of the result depend upon pre-analytic, analytic and post-analytic steps. Therefore to ensure accurate test results, it is now accepted that quality assurance, quality control and quality

\footnotetext{
Correspondence:

Dr. Badri Thapa

Genesis Laboratory and Research

Kathmandu, Nepal

Tel: 977-1-4426059

Email: badri_bishal@yahoo.com
}

assessment constitutes an integral part of all HIV diagnostic testing available globally. This is only possible through development of External Quality Assurance Scheme (EQAS) for HIVI AIDS testing. There exist three types of EQAS; proficiency testing, on site validation and retesting. Retesting as the means of EQAS is becoming popular these days in resource limited settings and geographically diverse countries where the whole blood can be collected in filter paper, dried at room temperature and then mailed through regular post to the organizing laboratory. ${ }^{1}$

The use of whole blood in a filter paper for screening phenylketonuria in infants date back to 
$1973^{2}$ and this technique was first evaluated for HIV-1 sero-prevalence study in pregnant women in 1988. ${ }^{3}$ Preparing of dried blood spot (DBS) is simple, doesn't need advance expertise, is cost effective and remains stable at room temperature for a month and for 2 years at $-20^{\circ} \mathrm{C} .1,{ }^{4}$ The DNA as well as proteins remain stable in the filter paper and is appropriate for testing HIV antibodies along with genome detection for diagnosis and viral load monitoring. $5,6,7$ The eluted whole blood from a DBS can be tested using ELISA and western blot and the use of rapid test kits (Unigold, Determine and Oraquick) has also been validated. ${ }^{8}$ The DBS could be an EQA material for HIV testing in resource limited country like Nepal. Hence, we have studied the feasibility of DBS as a material for HIV EQAS in Nepal.

\section{METHODOLOGY}

This study was conducted between October to December 2009. National Public Health laboratory (NPHL), Kathmandu was chosen as the organizing laboratory and 5 VCT centers from Central Development Region (Bharatpur Hospital (BH), Family Planning Association of Nepal (FPAN) and Mid-Western Region (Western regional Hospital (WRH), Nepal Red Cross Society (NRCS) and Walling Primary Health Care Center (WPHC)) were chosen as participating laboratories. These sites are run by government and non-government organizations and are located at different geographical locations. BH, FPAN, WHR, NRCS and Walling VCT center are at 155, 157, 200, 200 and 270 kilometers from NPHL, respectively.

\section{Sample collection, testing, storage and transportation from the participating laboratories}

With all aseptic precautions and fulfilling the norms of VCT centers, whole blood from the clients visiting the VCT centers was collected in two vials, with or without Ethylenediamine tetraacetic acid (EDTA). Thirty five paired samples (for onsite HIV testing and for preparation of DBS cards) were evaluated for this study. The serum was separated from nonEDTA vial for onsite HIV test using HIV rapid test kits following the National serial algorithm which is based on WHO/UNAIDS (Determine; test 1 , Unigold; test 2 and SD Bioloine; tie-breaker test). ${ }^{1}$ The samples were spotted in a DBS card from an EDTA whole blood following WHO guidelines. ${ }^{1}$ Two DBS cards for each sample were prepared. The DBS card was packed in a small zip-lock bag with some desiccants and was left at room temperature. Ten small zip-lock bags containing DBS cards were finally kept in bigger zip-lock bag along with the sheet mentioning the test result. One set was transported to NPHL immediately and the other was stored at room temperature and posted using normal postal service by the end of the month. The NPHL and Nepal government's criteria for transportation of bio-hazardous materials were met. Pre-analytical, analytical and post-analytical skills of the laboratory staff and space and equipment used in the laboratories were assessed through pre-evaluated questionnaire and checklist.

\section{At the participating laboratory, NPHL}

One staff was assigned for receiving, registering and storage of DBS card. The DBS card that was received at NPHL was checked for validity (condition of the DBS card, quality of spots, packing, any deterioration of desiccants and code number) and DBS cards with good quality were registered. Different code number for each DBS card was reassigned and was kept confidential. The cards were left at room temperature if the card was tested within 1 month of the preparation or was stored at -20C, if it was tested after 1 month. The DBS spots from the DBS cards were eluted as per the manufacturer's guidelines (SigmaEldrich, Germany). The eluent was tested using rapid test following the same algorithm followed at participating laboratories and was also tested using ELISA (HUMAN, Australia). Different person was assigned to test samples with different assays. Third person was assigned to manage the data. The results were compared between rapid test and ELISA and were also compared with the results that were obtained at the participating laboratory. The result was recorded in Excel spread sheet and was analyzed using Statistical Package for Social Science (SPSS) 15.0 for windows. 


\section{RESULTS}

Thirty five DBS cards were received at NPHL on 2, 1, 3, 3 days (mean, 2.25 days) (BH, FPAN, WHR and NRCS, respectively) using the normal postal delivery. Samples were not received from WPHC. These 35 posted DBS samples and those transported immediately to NPHL were of good quality and were finally processed. Concordant result was obtained between the two samples received immediately and by postal delivery using serial testing algorithm by rapid HIV test kits (Determine; test 1, Unigold; test 2, SD Bioline, tiebreaker test).

However, when these DBS eluted samples were tested with ELISA some discrepancies were seen. Reactive samples with rapid test were 17 (48.6\%) while $18(51.4 \%)$ were reactive with ELISA. Similarly, non-reactive samples with rapid test were 18 (51.4\%) and with ELISA were 17 (48.6\%). Thirty two samples were concordant between two assays and 3 samples were discordant (Table 1). Altogether, $32(91.4 \%)$ samples were concordant between two assays and $3(8.6 \%)$ samples were discordant.

Table 1. Number of DBS samples tested by Rapid test and ELISA

\begin{tabular}{|l|l|c|c|c|c|}
\hline \multirow{3}{*}{ Sample } & \multirow{2}{*}{ Result } & \multicolumn{4}{|c|}{ Assays } \\
\cline { 3 - 6 } & & \multicolumn{2}{|c|}{${ }^{*}$ RT } & \multicolumn{2}{c|}{ ELISA } \\
\cline { 3 - 6 } & & No. & $\%$ & No. & $\%$ \\
\hline DBS & Reactive & 17 & 48.6 & 18 & 51.4 \\
\hline & Nonreactive & 18 & 51.4 & 17 & 48.6 \\
\hline
\end{tabular}

*RT, Rapid Test

\begin{tabular}{|c|c|c|c|c|c|c|c|}
\hline $\begin{array}{l}\text { VCT } \\
\text { sites }\end{array}$ & $\begin{array}{l}\text { No. of } \\
\text { spec- } \\
\text { imen }\end{array}$ & $\begin{array}{c}\text { ELISA } \\
\text { positive }\end{array}$ & $\begin{array}{c}\text { Determine } \\
\text { HIV-1/2 } \\
\text { positive }\end{array}$ & $\begin{array}{c}\text { ELISA } \\
\text { negative }\end{array}$ & $\begin{array}{c}\text { Determine } \\
\text { HIV-1/2 } \\
\text { negative }\end{array}$ & FP & FN \\
\hline BH & 24 & 16 & 15 & 8 & 7 & 1 & 1 \\
\hline FPAN & 2 & 0 & 0 & 2 & 2 & 0 & 0 \\
\hline WRH & 5 & 1 & 1 & 4 & 4 & 0 & 0 \\
\hline NRCS & 4 & 1 & 0 & 3 & 4 & & 1 \\
\hline
\end{tabular}

FP, False positive; FN, False negative
Results obtained at FPAN and WRH were concordant with the results obtained at NPHL using both assays while results obtained at $\mathrm{BH}$ showed one false positive and one false negative result (Table 2). Similarly, one false negative result was obtained in sample obtained from NRCS.

Sensitivity of rapid test (Determine HIV-1/2) was determined using the reference assays, ELISA. Sensitivity was $88.9 \%$ and specificity was $94 \%$. The positive and negative predictive values were $94 \%$ and $88.9 \%$, respectively. Personal and infrastructural need of a laboratory and analytical skills of each staff was assessed using the preevaluated questionnaire and checklist. All the laboratories had enough well maintained space for performing rapid test and preparing a DBS card. Staff were adequately assigned for HIV testing and only some staff had formal training on rapid HIV testing. The lines of supervision were clear to the staff and supervisor checked the result before it was dispatched. Most of the equipments were present in all laboratories except stop watches and incinerator in some laboratories. The laboratory waste had been managed according to the biosafety rules. The micropipettes that had been used for testing were not calibrated in all laboratories. Although the staff were aware of DBS card, none of the staff had hands on practice with it.

\section{DISCUSSION}

The feasibility of DBS as a method of sample collection for NEQAS for HIV testing was studied. The DBS cards were transported using normal postal service and were found to be stable. Similar stability of a DBS card has been reported. We tested the DBS eluted sample using rapid test kit and ELISA. The rapid HIV test result showed $100 \%$ concordance using the serum and eluent from the DBS card. Similar results were obtained by Center for Disease control (CDC), Atlanta when using the rapid test kits on the DBS eluent but variation were seen among the test kits used. ${ }^{8}$ In this study, some discrepancies were noted when the DBS eluent was tested by ELISA. This might reflect some variations in the HIV-1 strains in Nepal and warrants for HIV-1 strain characterization in different risk 
groups and also for local manufacturing of a rapid test kit coated with antigens prevalent in Nepal. At the current situation where locally manufactured tests kits are not available, imported rapid test kits should be validated in the country before being used. The DBS eluent was assayed by ELISA kit, HUMAN, Australia. It would have been clear, if DBS eluent was also assayed by different ELISA kits as discrepancies has also been reported between different ELISA kits. ${ }^{9}$

The sensitivity and specificity of a Determine HIV$1 / 2$ rapid test were $88.9 \%$ and $94 \%$, respectively and the positive and negative predictive values were $94 \%$ and $88.9 \%$, respectively using ELISA as a reference method. Testing of DBS eluent using Determine HIV-1/2 rapid test gave sensitivity of 83$92 \%$ and specificity of $80-89 \% .{ }^{8}$ Although, WHO recommends test kits with $>95 \%$ sensitivity and specificity in HIV diagnostics, it is necessary to use a test kit having higher sensitivity and specificity. The sensitivity and specificity of HIV rapid test also depends on the HIV strains circulating in this region. The staff and equipments were sufficient to carry on the HIV NEQAS using a DBS card.

EQAS for HIV testing using a DBS sample is easy to collect and cost effective for resource limited settings. On top of this, DBS material could also be used for HIV-1 RNA and DNA PCR for diagnosis in infancy, HIV genotyping, HIV viral load monitoring and phenylketonuria and Palmodium falciparium diagnosis.2,10-12 HIV EQAS can also be performed with proficiency testing and onsite validation. Due to the laboratory infrastructural and geographical constraints, National EQAS using the proficiency panel (serum) is cumbersome as the specimen would take longer time to reach the corners of the country and is also almost impossible to maintain the cold chain and the integrity of the specimen during transportation. ${ }^{1}$ On site validation and correction of errors would be an idle choice but requires a team to travel to the participating laboratories regularly. This can become a hurdle when number of HIV tests/day is minimum and team would have to wait till the specimen arrives in the laboratory for on-site testing. The VCT centers studied were located around 270 kilometers (kms) and mean duration of postal delivery to NPHL was
2.25 days. Most of the VCT centers are located in major cities and these are located around 500-600 $\mathrm{kms}$ from NPHL and postal delivery will not take longer than 7-10 days. DBS is stable for one month at room temperature and can be safely delivered to NPHL from any VCT centers in the country. Further study is warranted to support this notion. Consideration of this method needs evaluation with larger sample size in wide geographical area. We could not include larger sample size representing laboratories located at different geographical areas as this study was a time bound study.

\section{CONCLUSIONS}

DBS is a simple and cost effective method for sample collection in a resource limited and geographic constrained country like Nepal. The DBS cards were easily posted, reached the laboratory with a mean duration of 2.25 days, sample was stable and the results obtained in all participating and organizing laboratories were concordant for most of the samples. Hence, DBS as a method of sample collection for HIV EQAS will offer a dynamic platform in Nepal. The spectrum of its use can also be extended for collection of samples for HIV surveillance, screening HIV in newborn, and study of HIV drug resistance.

\section{REFERENCES}

1. Guidelines for using HIV testing technologies in surveillance. UNAIDS/WHO working group on global HIVIAIDS/STI surveillance. 2001

2. Belton NR, Crombie JD, Robins SP, Stephen R, Farquhar JW. Measurement of phenylalanine in routine care of phenylketonuric children. Arch Dis Child 1973;48:472-5.

3. Parker SP, Cubitt WD. The use of the dried blood spot sample in epidemiology studies. J Clin Pathol 1999;52: 633-9.

4. Chaillet $P$, Zachariah R, Harries K, Rusanganwa $E$, Harries AD. Dried blood spots are a useful tool for quality assurance of rapid HIV testing in Kigali, Rwanda. Trop Med Hyg 2009;103:634-7

5. Sriwanthana B, Wetprasit N, Chareonsook S, Janejai N, Chareonsiriwatana W. A study to 
implement early diagnosis of HIV infection in infants born to HIV infection. Southeast Asian J Trop Med Public Health 2003; 34:221-6.

6. Alvarez-munoj MT, Zaragoza-Rodrı GS, RojasMontes O, Palacios-Saucedo G, Vazquez-Rosales G, Gomez- Delgado A, et al. High correlation of Human mmunodeficiency virus type 1 viral load measured in dried blood spot samples and plasma under different storage condition. Archives of Medical Res 2005;36:382-6.

7. Behets F, Kashamuka M, Pappaioanon, et al. Stability of human immunodeficiency virus type 1 antibodies in whole blood dried on a filter paper and stored under various tropical conditions in Kinshasa, Zaire. J Clin Microbiol 1992;1179-82.

8. Mei JV, Tanuri A, Rayfield M, Hannon WH. Use of dried blood spots with HIV rapid tests. Int Conf AIDS. 2004 Jul 11-16; 15: abstract no. B11204. Bangkok Thailand.

9. Cheingsong R, Gaolekwe S, Kalake $W$, et al. HIV-1 antibody testing of dried blood spot (whole blood): a preliminary field evaluation of HIV kits in Botswana. Int Conf AIDS 2002 Jul 7-12;14: abstract no. TuPeC4879.

10. Michaud V, Gil P, Kwiatek O, Prome S, Dixon $\mathrm{L}$, Romero L. Long-term storage at tropical temperature of dried-blood filter papers for detection and genotyping of RNA and DNA viruses by direct PCR. J Virol Methods 2007;146:257-65

11. Beck IA, Drennan KD, Melvin AJ, et al. Simple, sensitive, and specific detection of human immunodeficiency virus type 1 subtype B DNA in dried blood samples for diagnosis in infants in the field. J Clin Microbiol 2001;39:29-33.

12. Maenoa Y, Nakazawab S, Daoc LD, et al. A dried blood sample on filter paper is suitable for detecting Plasmodium falciparum gametocytes by reverse transcription polymerase chain reaction. Acta Tropica 2008;107:121-7. 